\title{
Design of Highly Birefringent and Low Confinement Loss Photonic Crystal Fibre by Introducing Asymmetric Defect Structures
}

\author{
Lukman V \\ Dept. of Electronics and Communication \\ Hindustan Institute of Technology and Science \\ Chennai, India
}

\author{
Jeena Maria Cherian \\ Dept. of Electronics and Communication \\ Hindustan Institute of Technology and Science \\ Chennai, India
}

\begin{abstract}
Photonic crystal fibres -micro structured fibre consisting of air hole arrays running along its length- have attracted much attention for fibre device application because of its unusual optical properties that are not realized in standard optical fibre, such as high birefringence, high nonlinearity, low confinement loss and tailorable chromatic dispersion. High birefringent PCF can be designed by breaking the circular symmetry and implementing asymmetric defect structures such as dissimilar air hole diameter, varying the number of circular and elliptical air holes.
\end{abstract}

This paper proposes a highly birefringent PCF with ultra-low confinement loss by introducing four ring solid core hexagonal structure which having both elliptical and circular air holes and introducing large air hole diameters near the core region for making the asymmetry. The modal birefringence, refractive indices, confinement loss and chromatic dispersion are calculated by using Finite element method (FEM). An endlessly single mode, high birefringent $\left(05.152 \times 10^{-3}\right)$ and a low confinement loss $\left(7.85 \times 10^{-5} \mathrm{~dB} / \mathrm{km}\right)$ found at the excitation wavelength of $\lambda=1550 \mathrm{~nm}$ with only four rings of air holes in the fiber cladding.

\section{General Terms}

Computer modelling, Data Communication

\section{Keywords}

Photonic crystal fibres, Birefringence, Confinement loss, Finite-Element Method (FEM)

\section{INTRODUCTION}

Photonic-crystal fiber (PCF); a new class of optical fiber has been very popular in the field of fiber-optic communications because of its confinement characteristics not possible in conventional optical fibers. The distinctive properties of PCFs are the high design flexibility [2], [8]. It is possible to obtain PCFs with diametrically opposite properties by changing the geometric characteristics of the air-holes in the fiber crosssection, that is, their dimension or position. The main difference between PCF and conventional fiber is the Photonic crystal fibers have an air-silica cross section, whereas standard optical fibers have all glass cross-sections [9].

According to their mechanism for confinement Photonic crystal fibers possess two modes of operation - index guiding and photonic band gap fibers - In index guiding PCF's, where light is confined in a higher refractive index region, the light is guided by total internal reflection between the solid core and cladding region. Instead, when the core has a refractive index lower than that of the cladding region, as in hollow-core fibers, it is necessary the presence of the photonic band gap (PBG) [1].

A PCF has a holey cladding region surrounding a solid core [1], [2], [4]. The birefringence and dispersive properties of Polarization maintaining photonic crystal fiber were analysed using Finite element method. To maintain the linier polarization high level of birefringence is required by reducing polarization mode dispersion. By breaking the circular symmetry and implementing asymmetric defect structures such as dissimilar air hole diameter, varying the number of circular and elliptical air holes, high birefringent PCF can be designed. The number of air hole rings around the central core need to be increased for making the structure more confined at a specific wavelength.[2],[10]. Model birefringence in this high birefringence PCF's has been reported to have values higher than that of conventional high birefringent fiber. By adding asymmetry along two orthogonal axes near the core region an effective index difference has created. This PCF structures provide ultra-low confinement loss and high birefringence with low dispersion and give single mode operation for very large wavelength range compared to the conventional fiber. The ratio of the hole-diameter to the hole-spacing pitch decides the various modes of operation that can be supported by the PCF structure.

This paper present a new cladding designs for high birefringence PCF with ultra-low confinement loss using finite element method. Two cases of PCF structure considered for comparison. The proposed structures have both elliptical and circular air holes with large air hole diameters near the core region for making the asymmetry.

For analysing and solving for equations with integrals and partial differentials we usually go for Finite element method. This method effectively eliminates the differential part or convert it into ordinary differential form which are then integrated by standard techniques like Euler's method, Runge kutta etc.

\section{ANALYSIS OF STRUCTURAL PROPERTIES}

\section{A. Birefringence}

A ray of light, during its passage through any anisotropic materials results in the splitting or decomposition of the ray into two. Commonly materials with uniaxial anisotropy-- have an axis of symmetry with no equivalent axis in the plane perpendicular to it-- exhibits this optical phenomenon. This axis of symmetry is termed as optical axis of a particular material. Light rays with linear polarizations in parallel and perpendicular direction will show unequal effective refractive 
indices $n_{\mathrm{e}}$ and $n_{\mathrm{o}}$ for extraordinary and ordinary emerging rays respectively. If an un-polarized beam of light enters a material with a nonzero acute angle to the optical axis, the perpendicularly polarized component will refract at an angle as per the standard law of refraction and its complementary component at a non-standard angle determined by the difference between the two effective refractive indices known as the birefringence magnitude.[2],[6]

$\Delta n=n_{e}-n_{o}$

The difference between the real part value of the effective indices of the pronounced fundamental core Eigen modes along $\mathrm{x}$ and $\mathrm{y}$ axis- $\mathrm{LP}_{01}{ }^{\mathrm{x}}$ and $\mathrm{LP}_{01}{ }^{\mathrm{y}}$

$\mathrm{B}=\left|\operatorname{Re}\left(\mathrm{n}^{\mathrm{x}}{ }_{\mathrm{eff}}\right)-\operatorname{Re}\left(\mathrm{n}^{\mathrm{y}}{ }_{\mathrm{eff}}\right)\right|$

\section{B. Confinement Loss}

The presence of finite air holes in the core region causes leakage of optical mode from inner core region to outer air holes is unavoidable which results in confinement losses. The confinement loss of the fundamental mode is calculated from the imaginary part of the complex effective index $\mathrm{n}_{\text {eff }}$, [1] using

Confinement loss $=(40 \pi / \ln (10) \lambda) \operatorname{Im}\left(\mathrm{n}_{\text {eff }}\right)[\mathrm{dB} / \mathrm{km}]$

where $\operatorname{Im}$ is the imaginary part of the neff

\section{Chromatic Dispersion}

The sum of material and waveguide dispersion gives the chromatic dispersion or total dispersion. The material dispersion is inherent to the material used to fabricate the fiber whereas the waveguide dispersion can be varied by changing the design parameter of the waveguide, thus allowing altering of the total dispersion.

The material dispersion can be neglected when $\mathrm{n}_{\mathrm{m}}(\lambda)$ becomes constant and the real part of the effective index of refraction $\mathrm{n}_{\mathrm{eff}}$ contains the dispersion information $\mathrm{D}$.

$$
\mathrm{D}=-(\lambda / \mathrm{c})\left(\mathrm{d}^{2} \operatorname{Re}\left(\mathrm{n}^{\mathrm{y}} \mathrm{eff}_{\mathrm{f}}\right) / \mathrm{d} \lambda^{2)}\right.
$$

Where $\mathrm{c}$ is the speed of light in vacuum and $\lambda$ is the operating wavelength.

\section{SIMULATION METHOD}

Curved Interface modeling of PCF is best and most accurately accomplished by means of full vectorial methods. Finite Element Method is the common among such approaches used for dielectric waveguide analysis. Analysis of PCF structures with differing hole-arrangements and shapes is possible. It is of great advantage in complex photonic crystal geometries and can be effectively employed for propagation and leaky modes and cavity modes for two dimensional Cartesian cross sections in cylindrical co-ordinates. [9]

The structural domain is particularly divided into triangular and quadrilateral sub domains whose refractive index profile can be clearly presented. For the purpose of modeling twodimensional PCF with finite geometry the use of anisotropic perfectly matched layers (PMLs) are important. This gives the flexibility for direct implementation of anisotropic PMLs parameters like dielectric permittivity, magnetic permeability and many more.

COMSOL Multiphysics [1],[6] is simulation software based on finite element analysis, suited for various physics and engineering applications. It offers high interfacing capabilities with MATLAB and its toolboxes for various programming, preprocessing and post processing steps. It also extends high range flexibility for dealing with partial differential equations (PDEs).

\section{HEXOGONAL DESIGN}

Hexagonal structure of PCF design is known to be the best arrangement for obtaining high birefringence, and comparatively low confinement loss. Here a highly birefringence hexagonal solid core PCFs with four rings was been modeled. The material used here is pure silica with refractive index 1.45. Two different structures were designed and compared for various parameters. The first structure suggests a four ring elliptical air holes with four large diameter circular air holes near the core whereas the second comprises four ring circular air holes with two elliptical hole near the core and two large diameter circular holes in the cladding. The key intension of such a design is to break the structural symmetry making a commendable effective index difference between two polarization modes lying orthogonal to each other and is been well analyzed by means of FV-FEM

Two structures were analyzed for the comparison of its structural properties. First case was proposed which consists of elliptical air holes with major and minor axis dimensions 1 $\mu \mathrm{m}$ and $1.4 \mu \mathrm{m}$ respectively and four large diameter circular air holes with diameter of $1.6 \mu \mathrm{m}$. the structure was analyzed for 2 varying pitch of $\Lambda=1.6 \mu \mathrm{m}$ and $\Lambda=2 \mu \mathrm{m}$. In second structure elliptical air holes are replaced by the circular air holes with diameter $1.2 \mu \mathrm{m}$ and in this two large elliptical air hole with dimensions a $1=1.2 \mu \mathrm{m}, \mathrm{b} 1=1.8 \mu \mathrm{m}$ are introduced near the core and two large circular air holes with diameter $1.82 \mu \mathrm{m}$ placed in the cladding. It was also analyzed and compared for the above given pitch values over $\mathrm{x}$ and $\mathrm{y}$ axis. All parameters were selected carefully to optimize optical results.

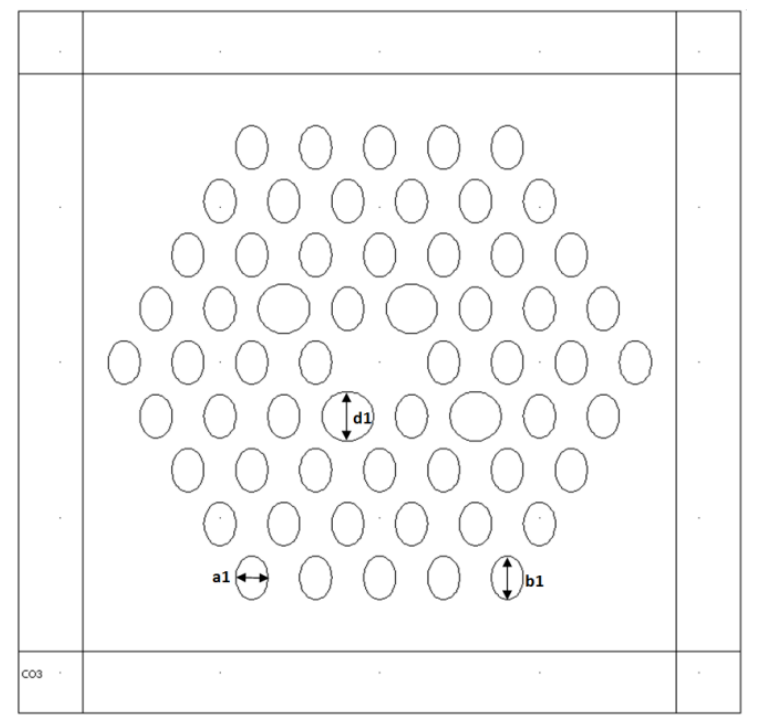

Fig 1. PCF structure case 1: (1) $\mathrm{a} 1=1 \mu \mathrm{m}, \mathrm{b} 1=1.4 \mu \mathrm{m}$, d1 $=1.6 \mu \mathrm{m} \quad \Lambda=1.6 \mu \mathrm{m}(2) \mathrm{a} 1=1 \mu \mathrm{m}, \mathrm{b} 1=1.4 \mu \mathrm{m}, \mathrm{d} 1=1.6 \mu \mathrm{m}$ $\Lambda=2 \mu \mathrm{m}$ 


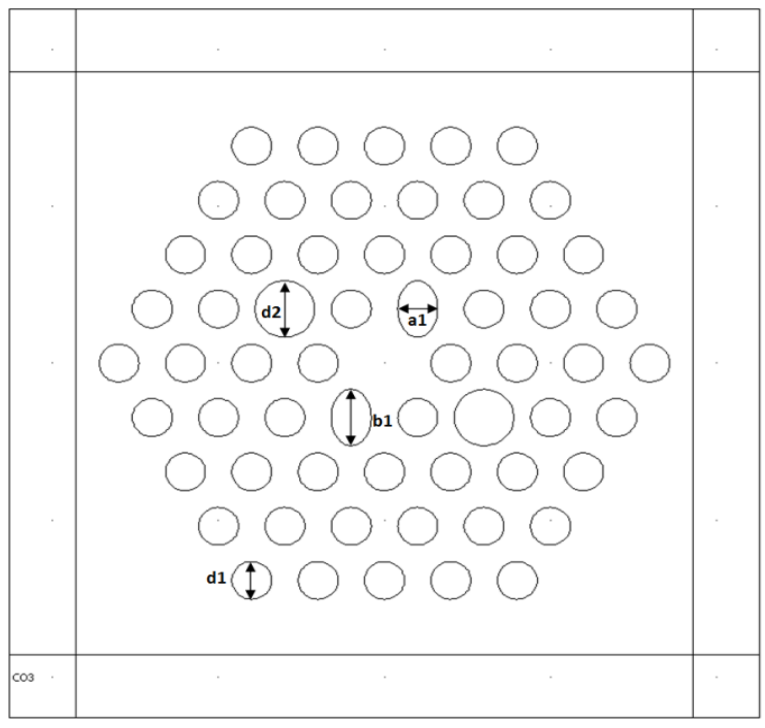

Fig 2. PCF structure case 2: (1) $\mathrm{d} 1=1.2 \mu \mathrm{m}, \mathrm{d} 2=1.8 \mu \mathrm{m}$, $\mathrm{a} 1=1.2 \mu \mathrm{m}, \mathrm{b} 1=1.8 \mu \mathrm{m}, \Lambda=1.6 \mu \mathrm{m},(2) \mathrm{d} 1=1.2 \mu \mathrm{m}$, $\mathrm{d} 2=1.8 \mu \mathrm{m}, \mathrm{a} 1=1.2 \mu \mathrm{m}, \mathrm{b} 1=1.8 \mu \mathrm{m}, \Lambda=2 \mu \mathrm{m}$.

The simulation is performed using COMSOL simulation tool to calculate the real effective refractive indices over different wavelengths of interest. The birefringence and confinement losses of each of the structure were formulated and analyzed.

\section{SIMULATION RESULTS}

Simulated the above modeled structures for differing pitch values with a defined PML thickness of $2 \mu \mathrm{m}$ using COMSOL simulating tool. The resultant $2 \mathrm{D}$ view of the simulation is given which shows high confinement of light beam defined. Highly birefringence structures are obtained in this single mode structure by slightly varying the defects as air holes diameter and shapes.

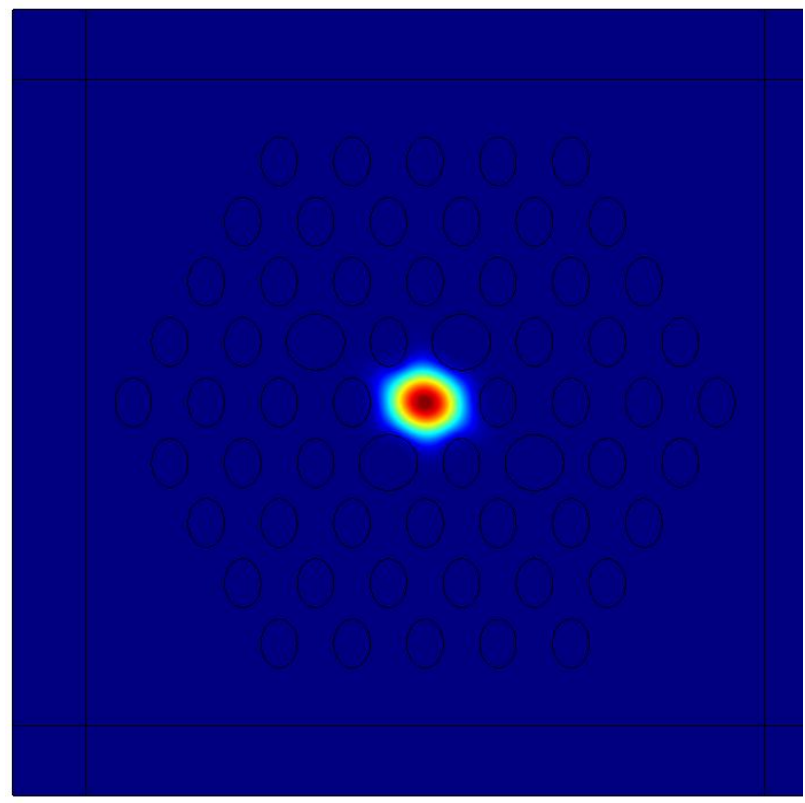

Fig 3. PCF structure with elliptical air holes a1 $=0.5 \mu \mathrm{m}, \mathrm{b} 1=0.7 \mu \mathrm{m}$, and Large diameter Circular air holes $\mathrm{d} 1=0.8 \mu \mathrm{m}$.
The simulated output produced highly confined single mode output as shown. By varying the pitch values produced slight variation in the structural properties..The structure with circular air holes and two large elliptical air holes near the cladding showed lowest confinment loss where as the first structure gives higher birefringence

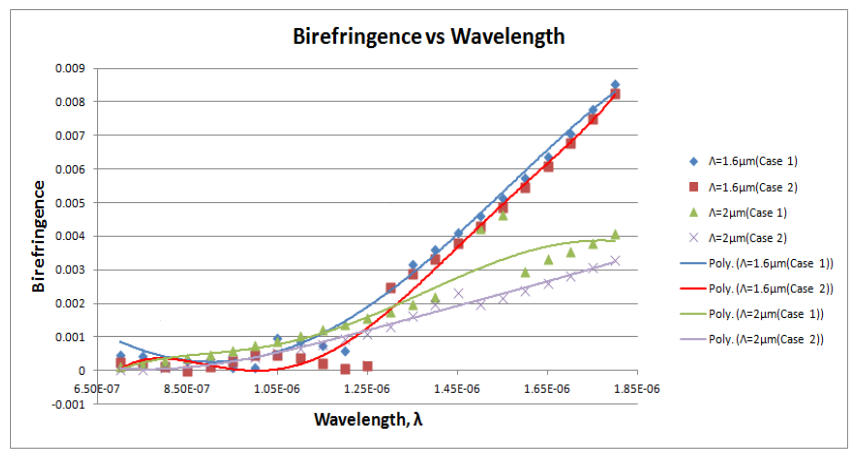

Fig 4. Birefringence vs Wavelength plot for four different cases considered

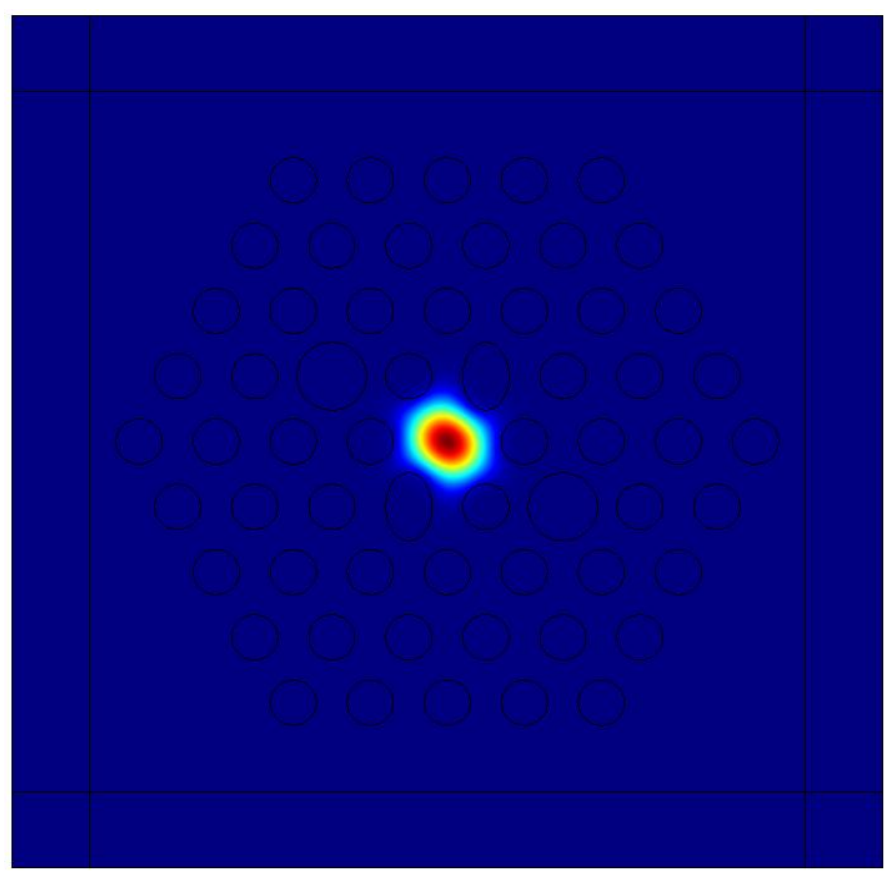

Fig 5. PCF structure with $a 1=2 \mu \mathrm{m}, \mathrm{b} 1=6.2 \mu \mathrm{m}$, $\mathrm{a} 2=0.328 \mu \mathrm{m}, \mathrm{b} 2=1.1 \mu \mathrm{m}$

The large air holes near the core provides the asymmetry and it significantly increase the birefringence and the large diameter circular air holes in the cladding region provide srong confinment ability. Confinement loss dincrases with decreesing pitch values for both the cases as shown also it incrasses with incrasing wavelength. The dispersion properties in both he structures has been compared and it shows that the dispersion is negative at lower wavelength and goes to positive and again decreas in higer wavelength. 


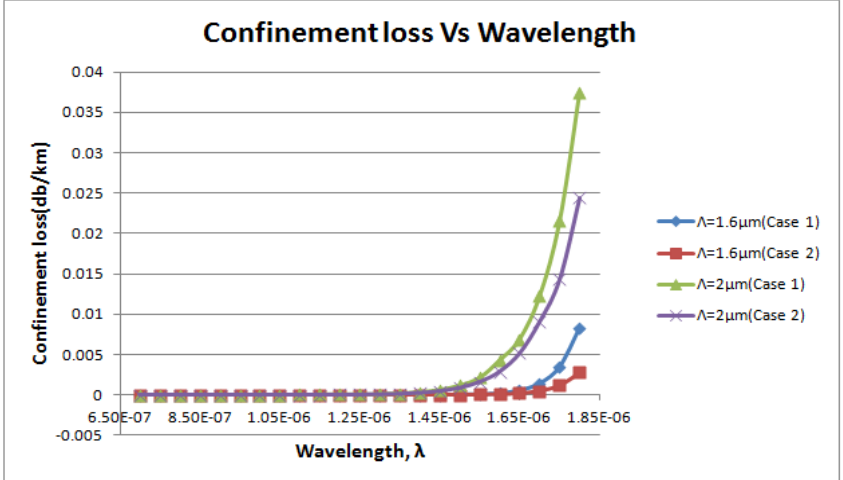

Fig 6. Confinement loss vs Wavelength plot for four different cases considered

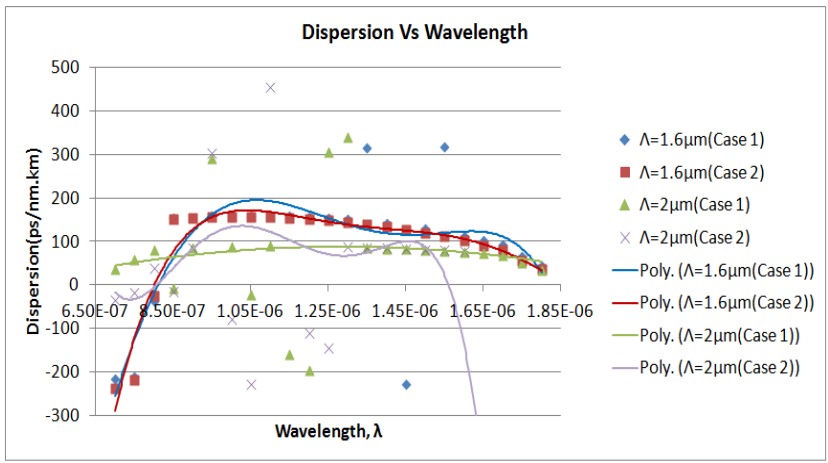

Fig 7. Dispersion Vs Wavelength plot for four different cases considered

\section{CONCLUSION}

Two structures of both circular and elliptical air holes with large diameter air holes near the core were modelled and simulated for different pitch values using a full-vector Finite element method with anisotropic perfectly matched boundary layer. It shown varying structural characterics like high birefrience low confinement loss and flattend chromatic dispersion.The large air holes near the cladding region provide asummetry in the core area and hence higer birefringence. Confinement loss shown its maximumum for pitch $\Lambda=1.6 \mu \mathrm{m}$ compared with that of $\Lambda=2 \mu \mathrm{m}$ for both the structures and getting endlessly single mode for a long range of wavelength. The highest birefringence obtained at the excitation wavelength $1550 \mathrm{~nm}$ is $05.152 * 10^{-3}$ with lowest confinement loss $7.85 \times 10^{-5} \mathrm{~dB} / \mathrm{km}$. These PCFs will have important applications in fiber communication and other sensor applications.

\section{REFERENCES}

[1] Ritu Sharma, Vijay Janyani, Anuradha Sharma, Design of Elliptical Air Hole PCF with Hybrid Square Lattice for High Birefringence and a Lower Zero Dispersion Wavelength International Journal of Computer Science \& Emerging Technologies (E-ISSN: 2044-6004) 238 Volume 2, Issue 2, April 2011

[2] Kuang-Yu Yang, Yuan-Fong Chau, Yao-Wei Huang, Hsiao-Yu Yeh,and Din Ping Tsai, Design of high birefringence and low confinement loss photonic crystal fibers with five rings hexagonal and octagonal symmetry air-holes in fiber cladding, JOURNAL OF APPLIED PHYSICS 109, 093103 (2011
[3] S. Sivabalan, Member, IEEE, and J. P. Raina,High Normal Dispersion and Large Mode AreaPhotonic Quasi-Crystal Fiber Stretcher, IEEE PHOTONICS TECHNOLOGY LETTERS, VOL. 23, NO. 16, AUGUST 15, 2011.

[4] Chuang Wu, Jie Li, Xinhuan Feng, Bai-Ou Guan, SideHole Photonic Crystal Fiber With Ultrahigh Polarimetric Pressure Sensitivity, JOURNAL OF LIGHTWAVE TECHNOLOGY, VOL. 29, NO. 7, APRIL 1, 2011

[5] Ya-Ni Zhang, Design and optimization of highbirefringence low-loss crystal fiber with two zerodispersion wavelengths for nonlinear effects, IEEE Journal of Quantum Electronics, Volume: 41 Issue:12,Dec 2005.

[6] Yuh-Sien SUN, Yuan-Fong CHAU_, Han-Hsuan YEH, and Din Ping TSAI, Highly Birefringent Index-Guiding Photonic Crystal Fiber with Squeezed Differently Sized Air-Holes in Cladding, Japanese Journal of Applied Physics Vol. 47, No. 5, 2008, pp. 3755-3759

[7] Yang Yue, Guiyun Kai, Zhi Wang, Yunfei Lu, Chunsh Zhang, Tingting Sun, Yan Li, Long Jin, Jianguo Liu,Yange Liu, Shuzhong Yuan, and Xiaoyi Dong Highly Birefringent Elliptical-Hole Photonic Crystal Fiber With Two Big Circular Air Holes Adjacent to core, IEEE PHOTONICS TECHNOLOGY LETTERS, VOL. 18, NO. 24, DECEMBER 15, 2006

[8] Soan Kim, Chul-Sik Kee, Chung Ghiu Lee, Hybrid Square-lattice Photonic Crystal Fiber by CLEO/Pacific Rim 2009.Shanghai, China.August 31-September 3,2009

[9] Vinod Kumar Singh, S. S. Mishra, Designing of Endlessly Single-Mode Highly Polarization Maintaining Birefringent Photonic Crystal Fiberwith Low Confinement Loss at Wavelength $1.55 \mu \mathrm{m}$

[10] Y. Yue, G. Y. Kai, Z. Wang, T. T. Sun, L. Jin, Y. F. Lu, et al, "Highly birefringent elliptical-hole photonic crystal fiber with squeezed hexagonal lattice," Opt. Lett., vol. 32, pp. 469-471, March 2007

[11] Zhang Ya-Ni, A Novel High-Birefringence High Nonlinear Negative-Dispersion Crystal Fiber with Square Mesh Cladding, 2011 International Conference on Electronics and Optoelectronics (ICEOE 2011).

[12] Peng Song, Yanrong Tong, Xinlian Chen, A Novel High Birefringence Photonic Crystal Fibre with Squeezed Elliptical Holes, 2010 3rd International Conference on Biomedical Engineering and Informatics (BMEI 2010).

[13] Lin An, Zheng Zheng, Zheng Li, Yang Liu, Tao Zhou and Jiangtao Cheng, Ultra-wideband Singlepolarization Single-mode Photonic Crystal Fiber with High Nonlinearity and Low Dispersion, 2009 OSA/ACP 2009. 